\title{
Benign neurogenic amyotrophy in Klinefelter's syndrome
}

\author{
S Matsubara, M Yoshino, M Takamori
}

\begin{abstract}
Two cases of benign neurogenic amyotrophy associated with Klinefelter's syndrome are reported. Both presented with slowly progressive, diffuse neurogenic muscle atrophy of juvenile onset. Both had a karyotype of XXY. Amplification, by the polymerase chain reaction, of a fragment of androgen receptor that was related to bulbospinal muscular atrophy, showed no abnormality. Treatment with androgen in one case provided no benefit. Benign neurogenic amyotrophy in the Klinefelter's syndrome is likely to be an independent type of motor neuron disease and suggests that the $X$ chromosome plays an important part in the biology of motor neurons.
\end{abstract}

$(\mathcal{F}$ Neurol Neurosurg Psychiatry 1994;57:640-642)

Motor neuron disease is a group of conditions, including amyotrophic lateral sclerosis, that involve upper and lower motor neurons. Besides studying amyotrophic lateral sclerosis itself, it is important to identify other conditions with motor neuron involvement, as they may enable us to gain new knowledge of the pathophysiology of the motor neurons. In this paper, two cases of benign neurogenic amyotrophy associated with Klinefelter's syndrome are reported. Only one similar case has been described. ${ }^{1}$

\section{Case reports}

Case 1

A man of 46 had been thin since childhood. At school, he was the slowest at running and never able to lift himself above a horizontal bar in the gymnasium. In his twenties and thirties, he was a manual worker and aware that despite being right handed, his right arm was thinner than his left. At the age of 40 , the weakness of his right arm became worse and caused him considerable handicap at work. Then he began to have difficulty in climbing stairs. Weakness of the limbs became aggravated in the subsequent years. After exercise he had painful stiffness of the legs. $\mathrm{He}$ noticed fine tremors in his arms. Other members of his family had no neurological disease.

On examination, he was a thin man of 162 $\mathrm{cm}$ height and $43 \mathrm{~kg}$ weight. He responded slowly but correctly to our questions on general knowledge. He had a mild degree of gynaecomastia and testicular atrophy. On neurological examination, the distal and proximal limb muscles and trunk and facial muscles were atrophied (fig 1a). The cranial nerves were normal apart from facial weakness. Knee jerks were barely elicitable; the other deep tendon reflexes were absent in all four limbs. He had fine hand tremors of 10 $\mathrm{Hz}$. The plantar responses were flexor bilaterally. His sensory system and coordination were normal.

Serum creatine kinase was raised to 518 IU/l (normal range for males 55-306 IU/1), of which $97.9 \%$ was $M M$ isoenzyme, the rest MB. An oral glucose tolerance test was normal. EMG of the biceps brachii and other muscles showed fibrillations at rest and giant neuromuscular units indicating active neurogenic atrophy. Motor nerve conduction velocities of the median, ulnar, tibial, and peroneal nerves were normal as were the sensory conduction velocities of the median, ulnar and sural nerves. Serum oestrone and oestradiol concentrations were 23.2 and 24.0 $\mathrm{pg} / \mathrm{ml}$ respectively and were both normal. Serum testosterone concentration was 249 $\mathrm{ng} / \mathrm{dl}$, slightly lower than the normal range (250-1100 ng/dl). Karyotype analysis revealed the XXY pattern.

He was treated each month with an intramuscular injection of testosterone enanthate $125 \mathrm{mg}$ for a year, but improvement in muscle strength did not occur.

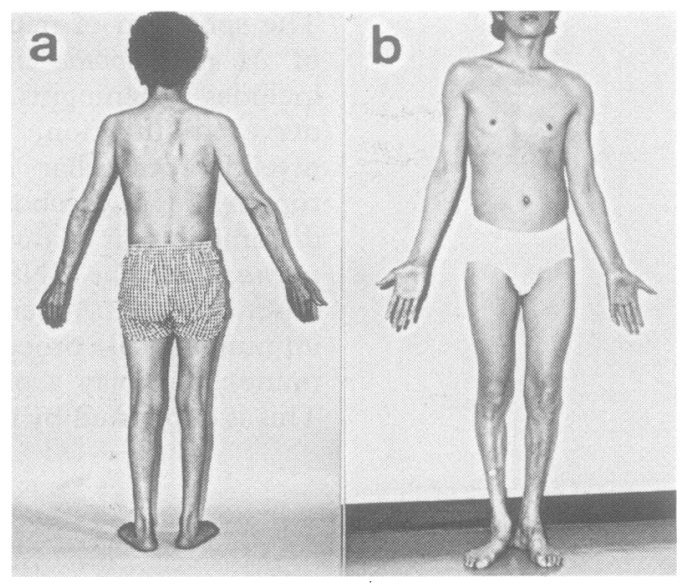

Figure 1 (a) case 1 ; (b) case 2. 
Case 2

A man of 29 had a waddling gait at the age of 10. He was mentally retarded to a mild degree. At school, he was unable to run or jump. Aged 17 he started to fall and often had cramps in his legs. Atrophy of the leg muscles was noted and he had an operation to elongate his left Achilles tendon. The weakness and atrophy of the leg muscles progressed gradually in the next 12 years. Before he attended our clinic, the neurologists at another hospital had made a diagnosis of amyotrophic lateral sclerosis.

He was a tall (height $179 \mathrm{~cm}$ ), thin (weight $58 \mathrm{~kg}$ ) man with a mild degree of gynaecomastia and testicular atrophy. His tongue was not atrophied. The jaw jerk was normal. All the leg muscles were atrophied and weak (fig 1b). There were no fasciculations. The legs were spastic and the knee and ankle jerks brisk. The plantar responses were extensor bilaterally. There was no sensory deficit or ataxia. On the Wechsler adult intelligence scale, his performance IQ was 93 , verbal IQ 69 , and total IQ 79 , all in the borderline range.

Serum creatine kinase activity, testosterone concentration, and other blood and cerebrospinal fluid tests were normal. His karyotype was XXY. The plain radiograph of the cervical vertebrae showed fusion of the vertebral bodies $\mathrm{C} 2$ and $\mathrm{C} 3$ without basilar impression. Myelography followed by CT myelography did not show stenosis of the spinal canal or any other abnormality.

The EMG of the anterior and posterior tibial muscles showed neuromuscular units of high amplitude and long duration indicating neurogenic processes. Motor and sensory nerve conduction velocites were normal.

\section{Histological investigations: both cases}

Muscle biopsies were taken from both cases. Frozen sections of $8 \mu \mathrm{m}$ were cut. They were stained with haematoxylin-eosin, Gomori trichrome, NADH-TR, periodic acid-Schiff reaction, and myosin ATPase ( $\mathrm{pH} \mathrm{4.2,} \mathrm{4.6,}$ and 9.4). Other portions of muscle were processed for electron microscopy. A sural nerve biopsy from case 2 was embedded in epoxyresin and examined by light and electron microscopy.

In the rectus femoris muscle of case 1, there were groups of small angulated fibres (fig 2a), which often harboured clusters of

Figure 2 (a) Muscle biopsy of case 1 . The rectus femoris muscle showed large groups of small angulated fibres indicating neurogenic muscle atrophy. neurogenic muscle atrophy. Peroneal muscle biopsy of case 2. Fibre type grouping with type 1 predominance was noted along with occasional small angulated fibres. Myosin ATPase activity ( $p H$ 9.4) (bar $=$ $100 \mu \mathrm{m})$. central nuclei. Hypertrophied fibres were frequent. NADH-TR activity showed fibre type grouping and occasional target fibres. Additional information was not obtained by electron microscopy.

The sural nerve of case 2 showed no abnormality. The peroneal muscle of case 2 showed occasional small angulated fibres and fibre type grouping, and a predominance of type 1 fibres (fig 2b). A histogram of fibre diameter, examined on sections stained with myosin ATPase ( $\mathrm{pH} \mathrm{9.4)} \mathrm{revealed} \mathrm{atrophied}$ fibres of both types 1 and 2 .

\section{Molecular biological investigations: both cases}

As the present cases had considerable similarity with bulbospinal muscular atrophy, ${ }^{2}$ the androgen receptor CAG repeat on the long arm of the $\mathrm{X}$ chromosome was amplified by the polymerase chain reaction. ${ }^{3}$ Normal controls and cases 1 and 2 showed products with a size of $405-450$ base pairs, whereas cases of bulbospinal muscular atrophy showed products of around 500 base pairs.

\section{Discussion}

Patients with Klinefelter's syndrome are described as having long slender extremities with poorly developed musculature. ${ }^{1}$ The present cases had weakness that disturbed their daily lives, clearly exceeding the mere tendency to poor general physique. Klinefelter's syndrome is associated with various neurological disturbances, including mental retardation, epilepsy, and essential tremor. In terms of neurogenic muscular atrophy, however, only a few cases have been reported and not all of them were fully investigated.

Case 2 of Nielsen ${ }^{1}$ was a man of 65 with diffuse muscle atrophy. The EMG and muscle biopsy showed neurogenic changes. Jenkins and Ferriman ${ }^{4}$ described a man of 42 with peroneal muscular atrophy. The nature of the atrophy was not clear.

The present cases and Nielsen's case $2^{1}$ had much in common. They had Klinefelter's syndrome and the karyotype XXY without any familial stigmata of muscle atrophy. They showed diffuse neurogenic muscular atrophy that progressed slowly over many years. There were a few points, however, which differed. Our case 2 had bilateral extensor plantar responses. The absence of cord compression on myelography does not preclude the possibility that the Klippel-Feil anomaly is responsible for it. Alternatively the upper and lower motor neurons may be affected by a common cause. Case 2 of Nielsen ${ }^{1}$ had a mild degree of sensory impairment in the legs.

The muscle biopsies in our cases showed changes typical of chronic neurogenic muscle atrophy. ${ }^{5}$ They include clusters of small angulated atrophic muscle fibres beside normal or often hypertrophic fibres. Fibre type grouping may be a sole abnormality in an early stage of neurogenic muscular atrophy. 
Absence of abnormality in the nerve conduction velocities and nerve biopsy and the occurrence of brisk tendon reflexes in case 2 are more in keeping with motor neuron disease than peripheral neuropathy.

Both of our present cases resembled bulbospinal muscular atrophy in regard to feminisation and neurogenic muscular atrophy and case 1 in regard to hand tremors. Bulbar symptoms, which are one of the hallmarks of bulbospinal muscular atrophy, were, however, absent. As all 20 Japanese cases of bulbospinal muscular atrophy that we have examined so far showed polymerase chain reaction products of abnormal length (unpublished data), the present cases are unlikely to have bulbospinal muscular atrophy.

The clinical features of the present cases and the case of Nielsen suggest that the involvement of motor neurons in Klinefelter's syndrome is a rare condition. It may be a fortuitous association of Klinefelter's syndrome and motor neuron disease. The clinical course of the present cases resembled that of hereditary motor neuron disease ${ }^{6}$ rather than sporadic amyotrophic lateral sclerosis, which is far commoner, yet none of our cases had a family history of motor neuron disease. These points accord with out hypothesis that motor neuron disease is related to Klinefelter's syndrome.

Androgens prevent loss of motor neurons in rats. ${ }^{7}$ Whether the presence of an extra $\mathrm{X}$ chromosome or androgen deficiency play a part in the motor neuron involvement remains to be elucidated.

We are grateful to Dr WGP Mair who encouraged us and kindly reviewed the English.

1 Nielsen J. Klinefelter's syndrome in a neurological ward. Acta Neurol Scand 1965;41:197-214.

2 Kennedy WR, Alter M, Sung JH. Progressive proximal spinal and bulbar muscular atrophy of late onset. A sexlinked recessive trait. Neurology 1968;18:671-80.

$3 \mathrm{La}$ Spada AR, Wilson EM, Lubahn DB, Harding AE, Fischbeck KH. Androgen receptor gene mutations in Xlinked spinal and bulbar muscular atrophy. Nature 1991;352:77-9.

4 Jenkins CO, Ferriman D. Klinefelter's syndrome associated with peroneal muscular atrophy. Proc $R$ Soc Med 1968;61:86.

5 Matsubara S, Tanabe H. A clinico-pathological study of chronic hereditary motor neuropathy. Acta Neuropathol chronic hereditary moto

6 Harding AE. Inherited neuronal atrophy and degeneration predominantly of lower motor neurons. In: Dyck PJ Thomas PK, Lambert EH, Bunge R, eds. Peripheral neuropathy, Vol 2. Philadelphia: WB Saunders, 1984; 1537-56.

$7 \mathrm{Yu}$ W-HA. Sex difference in neuronal loss induced by axotomy in the rat brain stem motor nuclei. Exp Neurol 1988;102:230-5. 